\title{
EDITORIAL
}

\section{Tackling trial troubles}

\author{
It is increasingly recognized that a key barrier to advancing cancer treatment is the time \\ and resources needed for the initiation and completion of clinical trials of anticancer drugs. \\ How might these challenges be addressed?
} Almost four decades after the 'war on cancer' began,
progress has been frustratingly slow, despite major
advances in our understanding of cancer biology. Among
the key contributing factors to this lack of progress are the
challenges of conducting cancer clinical trials, some of
which are highlighted in a recent article in the New York
Times. For example, according to the US ClinicalTrials.
gov registry, several thousand cancer clinical trials are
currently seeking patients. However, enrolling a sufficient
number of patients in a timely manner can be so difficult
that it prevents trial completion, to the detriment of both
the patients involved and the opportunity to advance
cancer treatment in general.

In part, this problem results from the small proportion of patients who enrol in trials - estimated at $\sim 3 \%$ of adult patients with cancer in the United States. This arises because of well-known barriers, including inconvenience and uncertainty for patients and disincentives for doctors. In addition to the costs for US oncologists associated with their patients participating in trials (for example, owing to loss of reimbursement fees), the burden of the bureaucratic requirements is often too large for small groups of physicians.

One possible approach to tackle the challenge is highlighted in the article ${ }^{1}$ : ensuring that the available resources are applied more efficiently, which could be achieved through wider use of adaptive clinical trial designs $^{2}$. In a traditional clinical trial, patients might be assigned to one of two drugs, and a decision about which is more effective is made when the trial is completed. By contrast, in an adaptive clinical trial, data can be used as they become available to modify the trial design. For example, the trial population in exploratory, early-stage trials can be progressively focused on patient subsets that are having the strongest response to treatment, allowing more efficient identification of the most appropriate patient population for confirmatory largescale trials.

A second approach - reducing the administrative burden that inhibits the initiation and completion of clinical trials - is described in a recent paper in the Journal of Clinical Oncology $y^{3}$. The effects of this burden have only recently been investigated, with initial studies indicating that it can take around 800 days from the concept stage for a cancer clinical trial to be initiated in the United States, with an average of 3-6 months elapsing just between approval of an investigational new drug (IND) application by the US FDA and entry of the first patient into a Phase I trial. As well as the overall slowing of the clinical trial process, it is possible that scientific advances made during this time may make the trial, or parts of it, outdated.

To address this issue, the authors of the recent paper ${ }^{3}$, who are all involved in an ongoing partnership between the M.D. Anderson Cancer Center in Texas, USA, and AstraZeneca, investigated the barriers to clinical trial initiation, with the aim of enrolling patients in a Phase I trial of a novel anticancer drug without a substantial delay following approval of an IND. Perhaps most importantly, tracing and re-examining the administrative processes, both at the academic centre and at the company, from the key convergence point of IND approval, identified steps that could be performed in parallel, rather than in series. Coupled with close communication at each stage, this ultimately allowed the first patient to be enrolled $\sim 48$ hours after IND approval and 46 days after the study protocol was finalized, compared with a typical time of $\sim 136$ days for this stage of the trial process.

The wave of targeted cancer drugs based on molecular knowledge that have emerged in recent years hold considerable promise for improving cancer treatment. However, with more possible treatment regimes to evaluate, and the potentially greater complexities of recruiting patients with more specific disease characteristics, delays in trial initiation and limited patient resources have the potential to become an increasingly substantial barrier to advancing the development of anticancer therapies. More widespread application of strategies to accelerate the administrative aspects of clinical trials, in concert with novel adaptive trial designs, might therefore play a key part in achieving long-awaited breakthroughs in the war on cancer.

\footnotetext{
. Kolata, G. Lack of study volunteers hobbles cancer fight. New York Times (New York) A1 (3 Aug 2009).

2. Berry, D. A. Bayesian clinical trials. Nature Rev. Drug Discov. 5, 27-36 (2006).

3. Kuzrock, R. et al. Project zero delay: a process for accelerating the activation of clinical trials. J. Clin. Oncol. 3 Aug 2009 (doi:10.1200/ JCO.2008.21.6093)
} 